\title{
Cooperative production under diminishing marginal returns: Interpreting fixed-path methods
}

\author{
by Justin LEROUX
}

Cahier de recherche $n^{\circ}$ IEA-06-10

October 2006

ISSN : 0825-8643

Copyright (C) 2006 HEC Montréal.

Tous droits réservés pour tous pays. Toute traduction ou toute reproduction sous quelque forme que ce soit est interdite. Les textes publiés dans la série des Cahiers de recherche HEC n'engagent que la responsabilité de leurs auteurs.

La publication de ce Cahier de recherche a été rendue possible grâce à des subventions d'aide à la publication et à la diffusion de la recherche provenant des fonds de l'École des HEC.

Direction de la recherche, HEC Montréal, 3000, chemin de la Côte-Sainte-Catherine, Montréal (Québec) Canada H3T 2 A7. 


\title{
Cooperative production under diminishing marginal returns: Interpreting fixed-path methods*
}

\author{
Justin Leroux ${ }^{\dagger}$
}

July 31, 2006

\footnotetext{
*I am extremely grateful for Hervé Moulin's invaluable guidance and endless patience. I am also thankful for conversations with Phil Curry, Eric Friedman, Simon Grant, Hideo Konishi, Bart Lipman and James Schummer and comments from participants of the Micro Theory seminar of Rice University as well as an anonymous referee for suggesting improvements on the structure of this article.

†Institut d'Économie Appliquée, HEC Montréal, 3000 chemin de la côte-Sainte-Catherine, Montréal, Québec H3T 2A7, Canada. E-mail: justin.leroux@hec.ca, Phone: (+1) 514-3406864 .
} 


\begin{abstract}
Fixed-path methods (FPMs) were introduced to manage situations where several individuals jointly operate a single technology (see [4]). In the production context, they consist in allocating marginal increments of output according to a proportions vector which changes along an arbitrary path. While very appealing from an incentives viewpoint under diminishing marginal returns, the asymmetry of these methods lacks solid economic interpretation.

We provide such an interpretation by considering a situation where the technology to be shared results from the aggregation of private production processes. We propose a group-strategyproof mechanism under which no single agent wishes to secede from the partnership: the inverse marginal product proportions mechanism. It is the only FPM satisfying autarkic individual rationality; its path is uniquely determined by the technological contributions of the agents.
\end{abstract}

Keywords: Autarky, incentive compatibility, cooperative production, surplus sharing, serial rule, path methods.

JEL classification numbers: C72, D23, D62, D71. 


\section{Introduction}

When several producers of a private good jointly supply input to a single production technology, the classical question of how to share the proceeds from their cooperation arises (see [7], [14], [18]). In this regard, Recent work by Friedman (see [4], [5]) introduces the interesting class of fixed-path methods (hereafter FPMs). These methods share total output by allocating marginal increments according to proportions given along an arbitrary path.

Specifically, if $F$ is the commonly owned one-input/one-output production function, one must determine the division of total output, $F\left(\sum_{i} x_{i}\right)$, among the individuals for every contribution vector $\left(x_{1}, \ldots, x_{n}\right)$. An FPM is parametrized by a continuous increasing path $\phi(t)$ of non-negative coordinates, where $\sum_{i} \phi_{i}(t)=t$. Imagine a production process taking place continuously through time. Each $\phi_{i}(t)$ corresponds to the rate at which individual $i$ supplies input over time, and the slope of the path at time $t$ determines the proportions of the marginal product allocated to each agent (at time $t$ ). When the contribution of an individual $i$ meets his supply level, $x_{i}$, that individual stops supplying input and leaves the production process with the output share she has secured so far. The sharing process resumes with the remaining agents sharing the left-over technology among themselves, and ends when the supply level of all individuals is met.

While FPMs are appealing because of their very strong incentives properties when the production technology exhibits diminishing marginal returns, little economic justification is given for their asymmetry. In particular, existing work makes no recommendation as to which path to choose. We provide such a recommendation for the case where agents are endowed with a private production technology and decide to combine their production possibilities in order to benefit from enhanced productivity. We show that under diminishing marginal returns there exists a unique FPM ensuring that no agent wishes to secede from

the cooperative and utilize her private technology on her own. Alternatively, we show that sharing a technology using an FPM amounts to attributing to each agent a (virtual) private production technology; this decomposition is uniquely defined by the path underlying the given FPM. 


\subsection{The partnership problem}

As mentioned above, our interpretation of FPMs relies on juxtaposing the single technology model with a situation where several producers of a common private good decide to pool their private production possibilities. In addition to technological contributions, each producer makes input (or labor) contributions to the cooperative. We assume that input is fully observable and transferable across technologies.

In a stylized version of the problem, each agent makes her privately owned machine (her technology) available to all the group-members and can supply labor to any machine. Practical examples include farmers pooling their land in a cooperative; here, land is technology and input can be labor or seeds to be planted. Examples of such cooperatives can also be found in the fishing sector ([17]) and in the plywood industry ([2]). A similar situation arises whenever a group of experts (e.g. lawyers, physicians, financial advisors, car salesmen, etc.) who can rank their clients in decreasing order of profitability decide to engage in a partnership; each agent's client base then amounts to a decreasingreturns technology. By pooling their clienteles, agents can reallocate their time or resources (the input) across the total pool of clients.

We assume that individual technologies are known to the planner and exhibit diminishing marginal returns. However, information about the leisure/consumption trade-off of the agents is private, potentially leaving room for misrepresentation. The first requirement is that production possibilities and input contributions be pooled efficiently. Under diminishing marginal returns, there is a unique efficient way to reallocate a given amount of input across the various technologies. ${ }^{1}$ Thus, the autarkic use of the production possibilities, where agent $i$ only supplies input to her own technology, can be Pareto-improved. The aggregate production function (of the individual technologies) summarizes these production opportunities.

While production efficiency is easily obtained, allocative efficiency cannot be achieved, precisely because the preferences of the agents are private information (see [8]). In particular, it follows that a standard market mechanism-which computes the competitive wage and the equilibrium amounts of labor and consumption for each agent-is manipulable; it would be in some agents' best interest to artificially restrict her labor-supply schedule in order to command a

\footnotetext{
${ }^{1}$ For instance, if machine 1 is always more productive than the others, productive efficiency requires that agents $2, \ldots, n$ work on machine 1 instead of their own.
} 
higher wage. ${ }^{2}$

In what follows we insist on strategyproofness while relaxing full efficiency in favor of the milder requirement of voluntary participation, i.e., every participant should be at least as well off under the pooling method as she would be by using her own technology by herself; we refer to this condition as autarkic individual rationality (or $\underline{A I R}$ ), a term introduced by Saijo ([13]) in the public good context.

We describe below a simple, group-strategyproof mechanism satisfying AIR to manage a partnership: the inverse marginal product proportions mechanism (or $\underline{I M P P}$ ). ${ }^{3}$ The main contribution of the paper (i.e., the interpretation of the asymmetry of FPMs mentioned in the beginning of the introduction) will arise upon noticing that the IMPP mechanism is the only FPM satisfying AIR.

\subsection{The IMPP mechanism}

For the sake of exposition, we describe the IMPP mechanism in the case of three agents (farmers) pooling their private capital (land). In Figure 1 are depicted the marginal product curves of each farmer's own land $\left(M P_{1}, M P_{2}\right.$, and $\left.M P_{3}\right)$. We assume for clarity that each agent's utility is quasilinear in the amount of profit, $y_{i}$, she receives: $u_{i}\left(x_{i}, y_{i}\right)=y_{i}-v_{i}\left(x_{i}\right)$, where $v_{i}\left(x_{i}\right)$ is agent $i$ 's disutility of supplying $x_{i}$ units of labor expressed in monetary terms. ${ }^{4}$

IMPP works as follows. Consider a dynamic production process where labor is allocated to land so that marginal product is equalized across fields at all times (but decreases over time). In the first stage, each agent works on her own land and reaps the fruit of her labor, like in autarky, until one agent decides to stop working. That agent then "leaves" the procedure with the output she has secured thus far, but her unused land is now available for agents 2 and 3 to utilize in addition to their own. In our example, agent 1 leaves the procedure after supplying $x_{1}$ units of labor: the intersection of her marginal disutility of effort $\left(v_{1}^{\prime}\right)$ with the marginal product of her own land $\left(M P_{1}\right)$ occurs "first", i.e. for a higher marginal product level than the other agents $\left(\lambda_{1}>\lambda_{2}, \lambda_{3}\right)$.

With agent 1 gone, her left-over capital is divided between agents 2 and 3 in proportion to the inverse marginal product of lands 2 and 3, respectively. For

\footnotetext{
${ }^{2}$ A larger-scale manipulation of the sort contributed to the collapse of the California energy market in 2001. Manipulation was successful even when as many as two hundred electricity providers were involved! See [12]. We thank Simon Grant for this observation.

${ }^{3}$ The mechanism we propose is actually Nash-implementable with unique equilibrium. In this model the corresponding direct revelation mechanism is then group-strategyproof; see Section 2 for a discussion of other, weaker interpretations of incentive compatibility.

${ }^{4}$ Our results hold on more general preference domains.
} 
any marginal product level, $\lambda<\lambda_{1}$, compare the amounts of labor which can be assigned to fields 2 and 3 before reaching the marginal product $\lambda$. Suppose, as in Figure 2, that twice as much labor can be supplied to field 2 as can be supplied to field 3: $2 \alpha$ and $\alpha$, respectively. In a sense, field 2 is twice as productive as field 3. Accordingly, agent 2 will be allowed to supply twice as much labor as agent 3 to field 1 at the margin $-\frac{2}{3} \beta$ and $\frac{1}{3} \beta$, respectively - and receive the corresponding marginal increment of output.

The procedure is carried out until another agent decides to stop working (in our example, agent 2 departs after having supplied $x_{2}$ units of labor, see Figure 3 ) and the remaining agent (agent 3) can then freely operate all three fields at will.

Clearly IMPP satisfies AIR: agent 1 is indifferent between autarky and taking part in the partnership while agents 2 and 3 effectively see their production possibilities enhanced by the "departure" of agent 1 . In addition, the mechanism is strategy-proof: just like in an ascending-price auction, it is a strictly dominant strategy for each agent to "drop out" when the (common) marginal product equals her (private) marginal disutility of effort.

\subsection{IMPP and the commons problem}

Upon noticing that the production possibilities of the cooperative are summarized by the aggregate production function, as stated in Section 1.1, it becomes clear that the IMPP mechanism can be applied to situations where a group of users jointly operate a single facility exhibiting diminishing marginal returns: the familiar commons problem.

If agents have equal rights to the facility, by assigning to each of them virtual property rights to " $1 / n^{\text {th }}$ of the facility" and by applying IMPP one obtains the output-sharing version of the well-known serial mechanism discussed in [11]. Thus, much better incentives are achieved than under, say, average- or marginalproduct pricing ${ }^{5}$.

Similarly, if agents do not have equal access to the common facility for exogenous reasons (e.g. social status), these differences can be taken into account by assigning unequal virtual production functions before applying IMPP. The corresponding sharing rule allocates marginal increments of input, and their corresponding increments of output, along a path in the input space of the

\footnotetext{
${ }^{5}$ We refer the reader to [11] for a discussion of the serial cost-sharing rule in comparison to average- and marginal-cost pricing.
} 
agents. The slope of this path describes each individual's relative access to the facility. After an agent's supply of input is met, the procedure carries on along the projection of the path onto the space of the remaining "active" agents. In other words, the active agents split the increments of input which would have been assigned to inactive agents (i.e. their "rights to using the facility") in the relative proportions granted by the original path. Such procedures are precisely the FPMs mentioned earlier and are presented formally in Section 3.

\subsection{Organization of the article}

After a brief review of some related literature, the remainder of the paper is organized in reverse order relative to the introduction. We first define FPMs in a single technology model (Section 3). We then show the close relationship between FPMs and the IMPP mechanism (Section 4). Finally, we recall the strong incentives properties of FPMs and discuss the appeal of the IMPP mechanism as a reasonable compromise between simplicity and responsiveness to capital contributions (Section 5). Most proofs can be found in the Appendix.

\section{Relation to the literature}

This work contributes to the large literature exploring the trade-off between efficiency and incentive compatibility in the production and distribution of private goods.

Our main result (Theorem 1) can be viewed as a follow-up on work by Friedman ([4], [5]). The FPMs mentioned in the introduction are the output-sharing version of the mechanisms introduced in [4] as non-anonymous generalizations of the Moulin and Shenker serial cost-sharing mechanism. Theorem 1 establishes that to each partnership problem (i.e. any profile of technological contributions) corresponds a unique FPM sharing the aggregate production function while satisfying AIR. Conversely, we show (Theorem 2) that to each FPM corresponds a unique (virtual) decomposition of the common facility into individual technological contributions; the FPM is then the IMPP mechanism applied to this very decomposition. We thus establish a bijection between the class of FPM to manage a commonly owned facility and possible distributions of property rights to the facility. This result provides economic justification for the non-anonymity of FPMs.

Many rules outside of the class of FPMs meet our high standards of incentive 
compatibility; their more complex path structure is similar to the "path functions" of Sprumont ([16]) (see Remark 2). This contrasts with Moulin ([10]) where it is shown in the discrete framework that FPMs - and the corresponding IMPP mechanisms - are in fact characterized by an incentive compatibility requirement fairly close to ours (see our discussion at the end of Section 3). This discrepancy illustrates a subtle difference between the discrete and continuous versions of the model and is worthy of future research.

Recent related literature on the common production of private goods considers weaker interpretations of incentive compatibility (see, e.g., [1], [15]). For instance, Corchón and Puy establish in [1] that any continuous sharing rule admits a Pareto-efficient allocation which can be Nash-implemented. Yet, any game implementing such an outcome must have several, non-welfare-equivalent Nash equilibria at some profiles. Our incentives compatibility criterion insists on the uniqueness of the Nash equilibrium, a much more demanding requirement than the above kind of Nash-implementability.

\section{$3 \quad$ The single technology model}

Let $N=\{1, \ldots, n\}$ be the set of agents. Let $F: \mathbb{R}_{+} \rightarrow \mathbb{R}_{+}$be a production function which is increasing, strictly concave such that $F(0)=0$. We denote by $\mathcal{F}$ the class of such functions. Each agent $i$ provides a non-negative amount $x_{i}$ of input to the common technology, and receive a non-negative quantity $y_{i}$ of output such that $\sum_{i} y_{i}=F\left(\sum_{i} x_{i}\right)$. We write $x=\left(x_{1}, \ldots, x_{n}\right)$ and for any $i \in N,\left(x_{i}^{\prime}, x_{-i}\right)$ is the vector of inputs where the $i$ th entry of $x$ has been replaced by $x_{i}^{\prime} \in \mathbb{R}_{+}$. A $\underline{\text { bundle }}$ is an element $z_{i}=\left(x_{i}, y_{i}\right) \in \mathbb{R}_{+} \times \mathbb{R}_{+}$; we define an allocation, $z$, to be a list of $n$ bundles, one for each agent. We denote by $Z_{F}=\left\{z \in\left(\mathbb{R}_{+} \times \mathbb{R}_{+}\right)^{N} \mid \sum_{i} y_{i} \leq F\left(\sum_{i} x_{i}\right)\right\}$ the set of feasible allocations under $F$.

Each agent $i$ can supply up to $M_{i}$ units of input (with $M_{i}$ possibly very large). Her preferences over bundles are defined on $\mathbb{R}_{+} \times \mathbb{R}_{+}$; they are continuous, convex, strictly increasing in $y_{i}$, strictly decreasing in $x_{i}$ and representable by a utility function $u_{i}$. While all our results are purely ordinal, we will use utility representations rather than the more cumbersome binary relation notation. We adopt the convention $u_{i}\left(x_{i}, y_{i}\right)=-\infty$ if $x_{i}>M_{i}$. We denote by $\mathcal{U}$ the class of preferences. A preference profile (or utility profile) is a list of $n$ preferences, $u=\left(u_{1}, \ldots, u_{n}\right) \in \overline{\mathcal{U}^{N}}$, one per agent. For any $j \in N$, we will sometimes abuse 
notations and write $u=\left(u_{j}, u_{-j}\right)$.

Definition 1 An F-sharing method (or F-sharing rule) is a differentiable mapping,

$$
\begin{aligned}
\xi: & \mathbb{R}_{+}^{N} \rightarrow \mathbb{R}_{+}^{N} \\
& x \mapsto\left(\xi_{1}(x), \ldots, \xi_{n}(x)\right) \quad \text { s.t. } \quad \sum_{i \in N} \xi_{i}(x)=F\left(\sum_{i \in N} x_{i}\right),
\end{aligned}
$$

which satisfies the following monotonicity property: $\frac{\partial \xi_{i}}{\partial x_{i}}>0$ for all $i \in N$.

We denote by $\mathcal{S}_{F}$ the class of $F$-sharing rules.

Monotonicity is a normatively appealing requirement. It states that an agent should receive strictly more output as her input contribution increases: it gives agents an incentive to supply input. Also, from the point of view of fairness, it implies that every agent will receive a positive fraction of the output resulting from her input contribution.

For any preference profile $u \in \mathcal{U}^{N}$ and any $F$-sharing method $\xi \in \mathcal{S}_{F}$, we denote by $G(\xi ; u)$ the game in which each agent's strategy space is $\mathbb{R}_{+}$and agent $i$ 's payoff is $u_{i}\left(x_{i}, \xi_{i}(x)\right)$ when $x_{j}$ is the strategy played by each agent $j \in N$.

We now define what we mean by "sharing a technology along a path". A path is a mapping

$$
\begin{aligned}
\phi: \quad & {\left[0, \sum_{j \in N} M_{j}\right] \rightarrow \times_{j \in N}\left[0, M_{j}\right] } \\
& t \mapsto\left(\phi_{1}(t), \ldots, \phi_{n}(t)\right)
\end{aligned}
$$

such that for all $i \in N$ the following two properties hold:

(a) $\phi_{i}$ is non-decreasing and therefore differentiable almost everywhere on $\mathbb{R}_{+}$ with respect to the Lebesgue measure,

(b) $\sum_{j} \phi_{j}(t)=t$ for any $t \in\left[0, \sum_{j} M_{j}\right]$.

We denote by $\mathcal{P}$ the class of paths.

Fix $\phi \in \mathcal{P}$. For any $i \in N$, define the mapping $\delta_{i}$ as follows:

$$
\begin{aligned}
\delta_{i}: \quad & {\left[0, M_{i}\right] \rightarrow \mathbb{R}_{+} } \\
& x_{i} \mapsto \min \left\{t \mid \phi_{i}(t) \geq x_{i}\right\}
\end{aligned}
$$

One interpretation is that if $t$ denotes time, $\delta_{i}\left(x_{i}\right)$ is the moment at which the $i^{\text {th }}$ coordinate along the path, $\phi_{i}(t)$, reaches the value $x_{i}$. Note that if $\phi_{i}$ is 
increasing, its inverse exists and $\delta_{i} \equiv \phi_{i}^{-1}$. If not, $\delta_{i}$ has a countable number of "jumps" correspondings to regions where $\phi_{i}$ is flat.

Given a path $\phi \in \mathcal{P}$, we define the fixed-path method generated by $\phi$, denoted $\xi^{\phi}$, as follows. Let $x \in \times_{i}\left[0, M_{i}\right]$, without loss we relabel the agents such that $\delta_{1}\left(x_{1}\right) \leq \delta_{2}\left(x_{2}\right) \leq \ldots \leq \delta_{n}\left(x_{n}\right)$; i.e. such that the coordinates of $x$ are met along $\phi$ in the natural order. Let $t \geq 0$ be such that $\phi(t) \leq x$, i.e. such that no agent's supply level has yet been met. $\xi^{\phi}$ recommends that the marginal product $F^{\prime}(t)$ be split between the agents according to the vector of proportions $\left(\phi_{1}^{\prime}(t), \ldots, \phi_{n}^{\prime}(t)\right)$ (recall that $\sum_{i} \phi_{i}^{\prime}(t)=1$ almost everywhere). Once the input supply of the first agent is met along the path $\left(\phi_{i}(t) \geq x_{i}\right)$, we freeze her output share and continue the sharing procedure with the remaining "active" agents. The subprocedure shares the remainder of $F$ along the projection of $\phi$ on the subspace $\left\{s \in \mathbb{R}_{+}^{n} \mid s_{1}=x_{1}\right\}$ until agent 2's supply is met. And so on. We next give a formal definition.

Definition 2 The fixed-path method generated by $\phi$, denoted $\xi^{\phi}$, is the $F$ sharing rule defined by:

$$
\begin{aligned}
& \xi_{1}^{\phi}(x)=\int_{0}^{\delta_{1}\left(x_{1}\right)} F^{\prime}(t) d \phi_{1}(t) \\
& \xi_{2}^{\phi}(x)=\int_{0}^{\delta_{1}\left(x_{1}\right)} F^{\prime}(t) d \phi_{2}(t)+\int_{\delta_{1}\left(x_{1}\right)}^{\delta_{2}\left(x_{2}\right)} F^{\prime}\left(x_{1}+\sum_{i \geq 2} \phi_{i}(t)\right) d \phi_{2}(t) \\
& \vdots \\
& \xi_{n}^{\phi}(x)=\int_{0}^{\delta_{1}\left(x_{1}\right)} F^{\prime}(t) d \phi_{n}(t)+\ldots+\int_{\delta_{n-1}\left(x_{n-1}\right)}^{\delta_{n}\left(x_{n}\right)} F^{\prime}\left(\sum_{i=1}^{n-1} x_{i}+\phi_{n}(t)\right) d \phi_{n}(t)
\end{aligned}
$$

for any $x \in \times_{i}\left[0, M_{i}\right]$.

A more compact notation is used by Friedman ([4], [5]): for any $i \in N$,

$$
\xi_{i}^{\phi}(x)=\int_{0}^{\infty} F^{\prime}(|\phi(t) \wedge x|) d\left(\phi_{i}(t) \wedge x_{i}\right)
$$

where $|\cdot|$ returns the sum of the coordinates of a vector and $\wedge$ is the componentwise minimum of two vectors.

It follows easily from the monotonicity of $F$ and the $\delta_{j}$ 's that $\xi^{\phi}$ is monotonic $\left(\frac{\partial \xi_{i}^{\phi}}{\partial x_{i}}>0\right.$ for all $i$ ); hence, $\xi^{\phi} \in \mathcal{S}_{F}$. Moreover, one can check (or see [4], Lemma 1) that $\xi_{i}^{\phi}$ is strictly concave in $x_{i}$.

We next give two examples of fixed-path methods:

Example 1: Incremental sharing. $(n=2)$ This method gives agent 1 full access to $F$; once she is served, agent 2 can use $F\left(x_{1}+\cdot\right)$ at will. The corresponding 
path is

$$
\phi^{I}: t \mapsto \begin{cases}(t, 0) & \text { if } t \leq M_{1}<+\infty \\ \left(M_{1}, t-M_{1}\right) & \text { if } M_{1} \leq t \leq M_{1}+M_{2}\end{cases}
$$

i.e., $\phi^{I}$ is a parametrization of the horizontal axis up to $x_{1}=M_{1}$. Output is awarded as follows:

$$
\left\{\begin{array}{l}
\xi_{1}^{\phi^{I}}(x)=F\left(x_{1}\right) \\
\xi_{2}^{\phi^{I}}(x)=F\left(x_{1}+x_{2}\right)-F\left(x_{1}\right)
\end{array}\right.
$$

Example 2: Weighted serial rule. Assume $M_{1}=M_{2}=+\infty^{6}$. Let $\alpha_{1}, \ldots, \alpha_{n}>$ 0 with $\sum_{i} \alpha_{i}=1$, and consider the path $\phi^{S}: t \mapsto\left(\alpha_{1} t, \ldots, \alpha_{n} t\right)$. Let $x \in \mathbb{R}_{+}^{N}$ and assume without loss that $\frac{x_{1}}{\alpha_{1}} \leq \frac{x_{2}}{\alpha_{2}} \leq \ldots \leq \frac{x_{n}}{\alpha_{n}}$. Expression (2) then yields:

$$
\xi_{i}^{\phi^{S}}(x)=\frac{\alpha_{i}}{\alpha^{i}} F\left(x^{i}\right)-\sum_{k=1}^{i-1} \frac{\alpha_{i} \alpha_{k}}{\alpha^{k} \alpha^{k+1}} F\left(x^{k}\right) \quad \text { for all } i=1, \ldots, n,
$$

where $\alpha^{k}=\sum_{j=k}^{n} \alpha_{j}$, and $x^{k}=x_{1}+\ldots+x_{k-1}+\frac{\alpha^{k}}{\alpha_{k}} x_{k}$. As a particular case, the usual serial rule assigns identical weight to each agent.

\section{Pooling private technologies}

We now give an interpretation of FPMs by establishing that sharing a single production technology according to an FPM amounts to attributing property rights on (possibly virtual) private technologies. Consider a situation where each agent privately owns a technology, $f_{i} \in \mathcal{F}$, which she decides to contribute to a cooperative along with an input level $x_{i} \in\left[0, M_{i}\right]$. One can think of the individual technologies as being machines (or capital) and of input as being labor time. Labor is observable and transferable, meaning that agents are able to work on machines other than their own. The manager of the partnership (the planner) allocates the labor time of the workers across the various machines; e.g., if $x_{1}=3$, agent 1 may be asked to spend, say, two units of input on machine 1 and one unit on machine 4 . The resulting total output is distributed between the agents according to their labor (the $x_{i}$ 's) and capital (the $f_{i}$ 's) contributions. Technologies are known to the planner, but the preferences of the agents are private information.

\footnotetext{
${ }^{6}$ Although $M_{1}$ and $M_{2}$ were originally defined as real numbers, the definition of the weighted serial rule readily extends to the case where they are infinite.
} 
Define $F^{*}$ to be the aggregated production function resulting from the efficient usage of the combined individual technologies:

$$
\forall t \in \mathbb{R}_{+} \quad F^{*}(t)=\max _{\substack{\left(x_{1}, \ldots, x_{n}\right) \in \times_{i \in N}\left[0, M_{i}\right] \\ \sum_{i} x_{i}=t}} \sum_{i=1}^{n} f_{i}\left(x_{i}\right) .
$$

Notice that because the $f_{i}$ 's belong to $\mathcal{F}, F^{*}$ must also belong to $\mathcal{F}$. We call $f=\left(f_{1}, \ldots, f_{n}\right) \in \mathcal{F}^{N}$ the technology profile (or capital profile).

Thus, the pooling framework is tantamount to the previous context of sharing a single technology. Here, however, autarkic individual rationality is a concern: no agent should be better off by using her private technology on her own. This voluntary participation requirement will end up determining uniquely the fixed-path method to use.

Definition 3 (AIR) An $f$-sharing method is an $F^{*}$-sharing rule $\xi$ such that for any preference profile $u$ and any Nash equilibrium $x^{*}$ of $G(\xi ; u)$ the following holds:

$$
u_{i}\left(x_{i}^{*}, \xi_{i}\left(x^{*}\right)\right) \geq s a_{i}\left(u_{i}\right) \equiv \max \left\{u_{i}\left(x_{i}, y_{i}\right) \mid y_{i} \leq f_{i}\left(x_{i}\right)\right\} \quad \forall i \in N .
$$

We say $\xi$ shares $f$ (or satisfies AIR with respect to $f$ ) and we denote by $\mathcal{S}_{f}$ the class of $f$-sharing methods.

Definition 4 Let $\phi^{*}$ be the mapping assigning to each $t \geq 0$ the unique solution vector of (3); $\phi^{*}$ is a path ${ }^{7}$. The inverse marginal product proportions (IMPP) mechanism is the FPM generated by $\phi^{*}$.

The following theorem motivates the use of the IMPP mechanism.

Theorem 1 The IMPP mechanism is the unique FPM satisfying AIR with respect to $f$.

The following comments concerning $\phi^{*}$ will prove useful. Because $\phi^{*}$ is the unique solution of expression (3), it follows that

$$
F^{* \prime}(t)=f_{i}^{\prime}\left(\phi_{i}^{*}(t)\right)
$$

\footnotetext{
${ }^{7}$ Note that concavity of the $f_{i}$ 's is necessary to ensure the uniqueness and monotonicity of the solution vector as well as its continuity with respect to $t$.
} 
whenever $\phi_{i}^{*}(t)>0$ (technology $i$ is in use). I.e., $\phi_{i}^{*}(t)$ is the level of input that can be assigned to technology $i$ before its productivity falls below $F^{* \prime}(t)$. Hence, for a given $t>0$, the larger $\phi_{i}^{*}(t)$, the more productive technology $i$ is.

We now give some intuition as to why $\xi^{\phi^{*}}$ not only satisfies AIR but also improves upon autarky. As long as all agents are active $\left(t \leq \min _{j} \delta_{j}^{*}\left(x_{j}\right)\right), \xi^{\phi^{*}}$ shares the marginal product $F^{* \prime}(t)$ according to the vector of ratios $\left(\phi_{1}^{* \prime}(t), \ldots, \phi_{n}^{* \prime}(t)\right)$. Hence, assuming for clarity that $\delta_{1}^{*}\left(x_{1}\right)$ is the smallest of the $\delta_{j}^{*}\left(x_{j}\right)$ 's, then

$$
\xi_{1}^{\phi^{*}}(x)=\int_{0}^{\delta_{1}^{*}\left(x_{1}\right)} F^{* \prime}(t) \phi_{1}^{* \prime}(t) d t=f_{1}\left(x_{1}\right)
$$

and agent 1 receives her stand-alone level of output. Now, for $\delta_{1}^{*}\left(x_{1}\right) \leq t \leq$ $\min _{j \neq 1} \delta_{j}^{*}\left(x_{j}\right), \xi^{\phi^{*}}$ shares the marginal output $F^{* \prime}(t)$ between agents $2, \ldots, n$ according to the ratios $\left(\phi_{2}^{* \prime}(t), \ldots, \phi_{n}^{* \prime}(t)\right) \times \frac{1}{\sum_{j>1} \phi_{j}^{* \prime}(t)}$. Clearly, for any $i \neq 1$, $\frac{\phi_{i}^{* \prime}(t)}{\sum_{j>1} \phi_{j}^{* \prime \prime}(t)} \geq \phi_{i}^{* \prime}(t)$ and agent $i$ receives no less (typically more) than her standalone share of output. And so on. Improvement upon autarky obtains by integration. In words, when an agent leaves the procedure what is left of her technology is shared between the remaining agents in proportion to their technological contributions to $F^{*}$. A formal proof of Theorem 1 can be found in Appendix A.1.

Remark 1 Among the rules generated by path structures as in [16], all those (and only those) whose main path is $\phi^{*}$ are $f$-sharing methods, but their subpaths may be arbitrary.

Theorem 1 has an interesting converse interpretation. Given a production function $F^{*}$, to any path $\phi^{*}$ corresponds a unique decomposition of $F^{*}$ into a "virtual" production profile, $f$, such that $\xi^{\phi^{*}}$ is the unique FPM sharing $f$.

Theorem 2 For any $F^{*} \in \mathcal{F}$ and any $\phi^{*} \in \mathcal{P}$, there exists a unique technology profile $f$ decomposing $F^{*}$ in the sense of (3) such that $\xi^{\phi^{*}}$ shares $f$. For any $i \in N, f_{i}$ is given by

$$
f_{i}\left(x_{i}\right)=\int_{0}^{x_{i}} F^{* \prime}\left(\delta_{i}^{*}(t)\right) d t
$$

for all $0 \leq x_{i} \leq M_{i}$, where $\delta_{i}^{*}$ is defined relative to $\phi_{i}^{*}$ as in expression (1).

Proof. Immediate from Theorem 1. Let $F^{*} \in \mathcal{F}, \phi^{*} \in \mathcal{P}$ and $f \in \mathcal{F}^{N}$ decomposing $F^{*}$ in the sense of (3) such that $\xi^{\phi^{*}}$ shares $f$. For any $i \in N$, 
expression (5) holds almost everywhere. I.e.,

$$
f_{i}^{\prime}(t)=F^{* \prime}\left(\delta_{i}^{*}(t)\right) \quad \text { almost everywhere }
$$

The result follows from integrating between 0 and $x_{i}\left(\right.$ recall $\left.f_{i}(0)=0\right)$.

To illustrate Theorem 2, we provide the virtual production profiles corresponding to examples of Section 3.

Example 1. $\xi^{\phi^{I}}$ gives priority to agent 1 . It is equivalent to sharing the production profile where agent 2's technology is useless compared to that of agent 1 on $\left[0, M_{1}+M_{2}\right]$.

Example 2. Agents contribute to $F^{*}$ in proportion to the $\alpha_{i}$ 's: $f_{i}(t)=$ $\alpha_{i} F^{*}\left(\frac{t}{\alpha_{i}}\right)$.

Theorems 1 and 2 together establish an interesting bijection between the family of FPMs and the possible distribution of property rights on $F^{*}$.

\section{Discussion}

The family of fixed-path methods (FPMs) was introduced in [4] as a nonanonymous generalization of the serial rule retaining its strong incentives properties:

Theorem 3 (Friedman) Let $\xi$ be an FPM, the following statements are true:

i) $G(\xi ; u)$ has a unique Nash equilibrium,

ii) every Nash equilibrium of $G(\xi ; u)$ is strong.

Proof. It is shown in [4] that for any production function $F \in \mathcal{F}$, any path $\phi \in \mathcal{P}$ and any preference profile $u \in \mathcal{U}^{N}$, the game induced by $\xi^{\phi}$ satisfies a more demanding equilibrium property called $O$-solvability.

It follows from a standard result of the implementation literature (see Theorem 7.2.3 in [3]) that the associated direct revelation mechanism is groupstrategyproof.

Remark 2 While Moulin and Shenker ([11]) established that the serial rule could be characterized by the equilibrium properties of Theorem 3 along with Anonymity $\left(x_{i}=x_{j} \Longrightarrow \xi_{i}(x)=\xi_{j}(x)\right)$, these properties do not characterize the class of FPMs when we relax Anonymity.

Proof. For instance, assume $n=3, F \in \mathcal{F}$ and let $\phi \in \mathcal{P}$. Consider an $F$ sharing rule $\xi$ which coincides with $\xi^{\phi}$ until one of the agents is served, say 
agent $i$, but then shares the remainder of $F$ between the remaining two agents along a strictly increasing subpath, $\psi\left(i, x_{i}\right)$, which depends on the identity of the first-served agent and her input supply level. Note that unlike for an FPM, $\psi\left(i, x_{i}\right)$ may differ from the projection of $\phi$ onto the plane $\left\{s \in \mathbb{R}_{+}^{n} \mid s_{i}=x_{i}\right\}$ for some pair $\left(i, x_{i}\right)$. It is clear that agent $i$ has the same unique dominant strategy under $\xi$ and under $\xi^{\phi}$. A straightforward application of Theorem 3 yields that the remaining agents also have a unique dominant strategy regardless of $\psi$. Yet, $\xi$ is not an FPM. These path structures are called path functions in [16], though his use of path functions is ultimately quite different from ours. ${ }^{8}$ Characterizing the class of strategy-proof mechanisms is still a very large open question and is beyond the scope of this paper. We refer the reader to a companion paper ([9]) for a two-agent characterization result.

In the discrete version of our model, Moulin ([10]) establishes that "incremental sharing rules" (the discrete equivalent of FPMs) are characterized by similar strategic properties for any number of agents. Interestingly, the continuous framework allows for a much richer class of incentive compatible rules.

We show on a straightforward example why some more complex rules do not meet our incentive compatibility requirement in the discrete setting. Consider a technology given by the discrete increments $\partial F: 4,2,1,0$ (i.e. $F(1)=4$, $F(2)=4+2, \ldots)$ to be shared between 3 agents, each of whom can supply 0 or 1 unit of input. Suppose that the path structure used to share $F$ gives priority to agent 1 and then gives precedence to agent 2 over agent 3 if $x_{1}=1$, but precedence to agent 3 over agent 2 if $x_{1}=0$. If preferences are such that agent 1 is indifferent between bundles $(1,4)$ and $(0,0)$, and if agent 2 prefers $(1,2)$ to $(0,0)$, then agent 1 can help out agent 3 by deciding not to work, thus giving her access to the bundle $(1,4)$ instead of $(1,1)$.

The above rule is immune to coalitional deviations in a weak sense: at least one agent in the deviating coalition does not strictly benefit (agent 1). Yet, not every Nash equilibrium of the supply game is strong due to agent 1's indifference between two bundles. Such indifferences are ruled out by the specifications of the continuous model.

As made clear in the previous section, the IMPP mechanism is essentially an FPM and, as such, meets high standards of incentive compatibility. Yet, sharing

\footnotetext{
${ }^{8}$ Note that when $n=2$, the type of methods just described cannot be distinguished from FPMs.
} 
rules outside the class of FPMs - like the ones generated by the path functions in [16] - also meets these standards, along with AIR. However, the specification of path functions can potentially be quite complex, requiring the specification of a subpath $\psi\left(i, x_{i}\right)$ for each agent $i$ at every level of input $x_{i}$, whereas the unique FPM satisfying AIR is entirely determined by the capital profile $\left(f_{1}, \ldots, f_{n}\right)$.

Admittedly, not all path functions need to be complex. Consider the rule coinciding with $\xi^{\phi^{*}}$ until the departure of agent 1 (subject to the usual relabeling of agents) and then giving full priority to agent 2 , then to agent 3 ; or the one sharing the remainder of the technology, $G(\cdot)=F\left(\cdot-x_{1}-\phi_{2}\left(\delta_{1}\left(x_{1}\right)\right)-\right.$ $\left.\phi_{3}\left(\delta_{1}\left(x_{1}\right)\right)\right)$, according to the (two-agent) Moulin and Shenker serial rule. From Remark 1, both these simple rules satisfy AIR. Yet, they are not responsive to capital contributions from the agents; in particular, they do not provide any incentives for the agents to supply capital, $f_{i}$, to the partnership. On the other hand, the IMPP mechanism rewards agents in proportion to the productivity of the technology contributed and thus encourages the supply of capital.

We contend that the IMPP mechanism is a reasonable compromise between simplicity and responsiveness to technological contributions, which are two appealing features for any practical profit-sharing mechanism in producer cooperatives or professional partnerships. In law firms, for instance, Gilson and Mnookin ([6], p. 370) express the difficulty of designing satisfactory productivity-based methods due to the fact that partners often negotiate their profit share by threatening to leave the partnership with their own client base (a maneuver called "grabbing"):

Because the ability to leave with the firm' clients is critical to the strength of a lawyer's threat, [...] lawyers have an important incentive to make sure that clients in fact remain theirs, and not the firm's $[\ldots]$.

We feel that the IMPP mechanism answers this calling for an incentive compatible mechanism which combines aspects of common ownership with the requirement that private property rights be respected. 


\section{A Proofs}

\section{A.1 Proof of Theorem 1}

Before proving Theorem 1, we present a lemma establishing that, under any FPM, $\xi^{\phi}$, any positive level of output, $x_{i}$, can be guaranteed at equilibrium by some preference $u_{i}^{*}$ for agent $i$. Its proof can be found in Appendix A.2.

Lemma 1 Let $\phi \in \mathcal{P}, i \in N$. For any $x_{i}>0$, there exists a preference $u_{i}^{*} \in \mathcal{U}$ such that the following holds:

$$
\forall u_{-i} \in \mathcal{U}^{N \backslash i} \quad x_{i}^{*}=x_{i}
$$

where $x^{*}$ denotes the unique Nash equilibrium of $G\left(\xi^{\phi} ; u_{i}^{*}, u_{-i}\right)$.

Now to the proof of Theorem 1. We first show that the IMPP mechanisms (i.e., $\xi^{\phi^{*}}$ ) satisfies AIR with respect to $f$. Fix any supply vector $\left(x_{1}, \ldots, x_{n}\right)$, and suppose, without loss of generality that $\delta_{1}^{*}\left(x_{1}\right) \leq \delta_{2}^{*}\left(x_{2}\right) \leq \ldots \leq \delta_{n}^{*}\left(x_{n}\right)$. It will suffice to show that the IMPP mechanism awards at least $f_{i}\left(x_{i}\right)$ to each agent $i$.

As it turns out, agent 1 receives exactly her stand-alone level of output:

$$
\xi_{1}^{\phi^{*}}(x)=\int_{0}^{\delta_{1}^{*}\left(x_{1}\right)} F^{* \prime}(t) \phi_{1}^{* \prime}(t) d t=f_{1}\left(x_{1}\right) .
$$

Agent 2 receives at least her stand-alone level of output:

$$
\begin{array}{r}
\xi_{2}^{\phi^{*}}(x)=\int_{0}^{\delta_{1}^{*}\left(x_{1}\right)} F^{* \prime}(t) \phi_{2}^{* \prime}(t) d t+\int_{\delta_{1}^{*}\left(x_{1}\right)}^{\delta_{2}^{*}\left(x_{2}\right)} F^{* \prime}(t) \frac{\phi_{2}^{* \prime}(t)}{\sum_{j \geq 2} \phi_{j}^{* \prime}(t)} d t \\
\geq \int_{0}^{\delta_{2}^{*}\left(x_{2}\right)} F^{* \prime}(t) \phi_{2}^{* \prime}(t) d t=f_{2}\left(x_{2}\right)
\end{array}
$$

Similarly, $\xi_{3}^{\phi^{*}}(x) \geq f_{3}\left(x_{3}\right)$, and so on.

Now for the other direction. Let $\phi \in \mathcal{P}$ such that $\xi^{\phi}$ shares $f$. For the rest of the proof we will write $F$ instead of $F^{*}$ as no confusion is possible.

Fix $x \in \times_{i}\left[0, M_{i}\right]$ such that $\delta_{i}^{*}\left(x_{i}\right)=\delta_{j}^{*}\left(x_{j}\right)$ for all $i, j \in N$; i.e. $x$ is a point on the graph of $\phi^{*}$. From Lemma 1 , there exists a preference profile $u \in \mathcal{U}^{N}$ such that $x$ is the unique Nash equilibrium of $G\left(\xi^{\phi} ; u\right)$. It follows that $\xi^{\phi}$ satisfies 
AIR with respect to $f$ only if for any $i \in N$ and any $x_{i}>0$ the following holds:

$$
\int_{0}^{\delta_{i}\left(x_{i}\right)} F^{\prime}(t) d \phi_{i}(t) \geq \int_{0}^{x_{i}} f_{i}^{\prime}(t) d t .
$$

The calculations of the integral on the left-hand side amounts to considering only the values of $t$ where $\phi_{i}$ is increasing (outside of these regions $d \phi_{i}(t)$ equals zero). On this restricted domain, $\delta_{i} \equiv \phi_{i}^{-1}$. Hence, it follows from (5):

$$
\int_{0}^{\delta_{i}\left(x_{i}\right)} F^{\prime}(t) d \phi_{i}(t)=\int_{0}^{x_{i}} F^{\prime}\left(\delta_{i}(t)\right) d t
$$

Similarly, the change of variable $t \rightarrow \phi_{i}^{*}(t)$ applied to the term on the right-hand side yields:

$$
\int_{0}^{x_{i}} f_{i}^{\prime}(t) d t=\int_{0}^{\delta_{i}^{*}\left(x_{i}\right)} f_{i}^{\prime}\left(\phi_{i}^{*}(t)\right) d \phi_{i}(t)=\int_{0}^{\delta_{i}^{*}\left(x_{i}\right)} F^{* \prime}(t) d \phi_{i}^{*}(t) .
$$

Therefore,

$$
\int_{0}^{x_{i}} F^{\prime}\left(\delta_{i}(t)\right) d t \geq \int_{0}^{x_{i}} F^{\prime}\left(\delta_{i}^{*}(t)\right) d t
$$

for all $i \in N$ and all $x_{i}>0$.

Let $i \in N$ and define $H_{i}\left(x_{i}\right)=\int_{0}^{x_{i}} F^{\prime}\left(\delta_{i}(t)\right) d t$ for any $x_{i} \geq 0 ; H_{i}$ is strictly increasing and strictly concave. Hence,

$$
\begin{aligned}
H_{i}\left(x_{i}\right) & \leq H_{i}\left(\phi_{i} \circ \delta_{i}^{*}\left(x_{i}\right)\right)+H_{i}^{\prime}\left(\phi_{i} \circ \delta_{i}^{*}\left(x_{i}\right)\right) \cdot\left(x_{i}-\phi_{i} \circ \delta_{i}^{*}\left(x_{i}\right)\right) \\
\text { i.e. } \quad H_{i}\left(x_{i}\right) & \leq H_{i}\left(\phi_{i} \circ \delta_{i}^{*}\left(x_{i}\right)\right)+F^{\prime}\left(\delta_{i}^{*}\left(x_{i}\right)\right) \cdot\left(x_{i}-\phi_{i} \circ \delta_{i}^{*}\left(x_{i}\right)\right)
\end{aligned}
$$

with equality if and only if $x_{i}=\phi_{i} \circ \delta_{i}^{*}\left(x_{i}\right)$. It follows from equations (8) and (9) that

$$
\begin{array}{ll} 
& \int_{0}^{x_{i}} F^{\prime}\left(\delta_{i}^{*}(t)\right) d t \leq \int_{0}^{\phi_{i} \circ \delta_{i}^{*}\left(x_{i}\right)} F^{\prime}\left(\delta_{i}(t)\right) d t+F^{\prime}\left(\delta_{i}^{*}\left(x_{i}\right)\right) \cdot\left(x_{i}-\phi_{i} \circ \delta_{i}^{*}\left(x_{i}\right)\right) \\
\Longleftrightarrow \quad \int_{0}^{x_{i}} F^{\prime}\left(\delta_{i}^{*}(t)\right) d t \leq \int_{0}^{\delta_{i}^{*}\left(x_{i}\right)} F^{\prime}(t) d \phi_{i}(t)+F^{\prime}\left(\delta_{i}^{*}\left(x_{i}\right)\right) \cdot\left(x_{i}-\phi_{i} \circ \delta_{i}^{*}\left(x_{i}\right)\right) \\
\Longleftrightarrow \quad \int_{0}^{x_{i}} F^{\prime}\left(\delta_{i}^{*}(t)\right) d t \leq-\int_{0}^{\delta_{i}^{*}\left(x_{i}\right)} \phi_{i}(t) F^{\prime \prime}(t) d t+F^{\prime}\left(\delta_{i}^{*}\left(x_{i}\right)\right) \cdot x_{i}
\end{array}
$$

the last expression is obtained by integrating by parts. Rearranging yields:

$$
\int_{0}^{\delta_{i}^{*}\left(x_{i}\right)} \phi_{i}(t) F^{\prime \prime}(t) d t \leq F^{\prime}\left(\delta_{i}^{*}\left(x_{i}\right)\right) \cdot x_{i}-\int_{0}^{x_{i}} F^{\prime}\left(\delta_{i}^{*}(t)\right) d t .
$$


Recall that $\delta_{i}^{*}\left(x_{i}\right)=\delta_{j}^{*}\left(x_{j}\right)$ for all $i \in N$; and write $z=\delta_{i}^{*}\left(x_{i}\right)$ for any $i$. Summing up over all $i \in N$ and using the fact that $\sum_{i} \phi_{i}(t)=t$ for any $t \geq 0$ and $\sum_{i} x_{i}=\sum_{i} \phi_{i}^{*}(z)=z$, we get:

$$
\begin{aligned}
& \int_{0}^{z} t F^{\prime \prime}(t) d t \leq F^{\prime}(z) \cdot z-\sum_{i=1}^{n} \int_{0}^{\phi_{i}^{*}(z)} F^{\prime}\left(\delta_{i}^{*}(t)\right) d t \\
& \Longleftrightarrow \quad \int_{0}^{z} t F^{\prime \prime}(t) d t \leq F^{\prime}(z) \cdot z-\sum_{i=1}^{n} \int_{0}^{z} F^{\prime}(t) d \phi_{i}^{*}(t)
\end{aligned}
$$

From $\sum_{i} \phi_{i}^{*}(t)=t$ and integrating by parts again, this yields an equality. Therefore, equation (8) must be an equality for all $i \in N$. The choice of $j$ and $x_{j}$ being arbitrary, it follows that $\delta_{i}\left(x_{i}\right)=\delta_{i}^{*}\left(x_{i}\right)$ for all $x_{i} \in\left[0, M_{i}\right]$ and for all $i \in N$. That is to say that $\phi_{i} \equiv \phi_{i}^{*}$ for all $i \in N$, proving the theorem.

\section{A.2 Proof of Lemma 1}

Notation: We fix a production function $F \in \mathcal{F}$, a path $\phi \in \mathcal{P}$ and a preference profile $u \in \mathcal{U}^{N}$. As no confusion may arise, we shall write $\xi$ instead of $\xi^{\phi}$. We denote by $F_{-}^{\prime}$ (resp. $F_{+}^{\prime}$ ) the left (resp. right) derivative of $F$. Similarly, $\frac{\partial^{-}}{\partial \lambda}$ (resp. $\frac{\partial^{+}}{\partial \lambda}$ ) is the left-derivative (resp. right-derivative) operator. Also, we write:

(i) $\delta\left(x_{1}, \ldots, x_{n}\right)=\left(\delta_{1}\left(x_{1}\right), \delta_{2}\left(x_{2}\right), \ldots, \delta_{n}\left(x_{n}\right)\right)$ for any $x \in \times_{i \in N}\left[0, M_{i}\right]$,

(ii) $\left(t_{1}, t_{2}, \ldots, t_{i-1}, t_{i} \cdot(n-i)\right)$ is the vector of $\mathbb{R}_{+}^{N}$ with the last $(n-i)$ coordinates equal to $t_{i}$,

(iii) for any $\left(t_{1}, \ldots, t_{n}\right) \in \mathbb{R}_{+}^{N}, \phi\left(t_{1}, \ldots, t_{n}\right)=\left(\phi_{1}\left(t_{1}\right), \phi_{2}\left(t_{2}\right), \ldots, \phi_{n}\left(t_{n}\right)\right)$ with a slight abuse of notation.

Let $i \in N$ and $x_{i}>0$. Consider a preference (utility) $u_{i}^{*}$ which is quasi-linear with respect to $y_{i}$ such that its indifference curves are piecewise linear with a single kink at $\left(x_{i}, y_{i}\right)$ for any $y_{i} \in \mathbb{R}$. Set the slope of these indifference curves to be no greater than $F_{-}^{\prime}\left(\delta_{i}\left(x_{i}\right)\right)$ before $x_{i}$ and no smaller than $F_{+}^{\prime}\left(x_{i}\right)$ after $x_{i}$; where "before $x_{i}$ " (resp. "after $x_{i}$ ") stands for "at any point of $\mathbb{R}_{+} \times \mathbb{R}$ with first coordinate smaller (resp. greater) than $x_{i}$ ".

We show below that the former quantity is the smallest variation in output that agent $i$ can obtain via $\xi$ by deviating infinitesimally from $x_{i}$ : it corresponds to the case where she is the first one served along the path (i.e., the agent with smallest $\left.\delta_{j}\left(x_{j}\right)\right)$. On the other hand, $F_{+}^{\prime}\left(x_{i}\right)$ is the largest variation in output obtainable via $\xi$ at $x_{i}$ by deviating marginally from $x_{i}$; it corresponds to the case where she receives all the output up to $F\left(x_{i}\right)\left(\delta_{j}\left(x_{j}\right)=0\right.$ for all $\left.j \neq i\right)$. 
Indeed, let $x_{-i} \in \mathbb{R}_{+}^{N \backslash i}$; then, from the definition of $\xi$, and keeping in mind that $|\cdot|$ returns the sum of the coordinates of a vector and $\wedge$ is the componentwise minimum of two vectors,

$$
\frac{\partial^{-}}{\partial \lambda} \xi_{i}\left(\lambda, x_{-i}\right)=F_{-}^{\prime}\left(\left|\left(\lambda, x_{-i}\right) \wedge \phi\left(\delta_{i}(\lambda) \cdot n\right)\right|\right) \quad \text { and } \quad \frac{\partial^{+}}{\partial \lambda} \xi_{i}\left(\lambda, x_{-i}\right)=F_{+}^{\prime}\left(\left|\left(\lambda, x_{-i}\right) \wedge \phi\left(\delta_{i}(\lambda) \cdot n\right)\right|\right)
$$

As the $i$ th component of both vectors $x$ and $\phi\left(\delta_{i}\left(x_{i}\right) \cdot n\right)$ is equal to $x_{i}$, the concavity of $F$ yields $F_{+}^{\prime}\left(\left|x \wedge \phi\left(\delta_{i}\left(x_{i}\right) \cdot n\right)\right|\right) \leq F_{+}^{\prime}\left(x_{i}\right)$. Moreover, the concavity of $F$ also yields $F_{-}^{\prime}\left(\left|x \wedge \phi\left(\delta_{i}\left(x_{i}\right) \cdot n\right)\right|\right) \geq F_{-}^{\prime}\left(\left|\phi\left(\delta_{i}\left(x_{i}\right) \cdot n\right)\right|\right)$; notice that this last term equals $F_{-}^{\prime}\left(\delta_{i}\left(x_{i}\right)\right)$. It follows from these two facts that:

$$
\left.\frac{\partial^{-}}{\partial \lambda} \xi_{i}\left(\lambda, x_{-i}\right)\right|_{\lambda=x_{i}} \geq F_{-}^{\prime}\left(\delta_{i}\left(x_{i}\right)\right) \quad \text { and }\left.\quad \frac{\partial^{+}}{\partial \lambda} \xi_{i}\left(\lambda, x_{-i}\right)\right|_{\lambda=x_{i}} \leq F_{+}^{\prime}\left(x_{i}\right)
$$

Hence, for any $x_{-i} \in \mathbb{R}_{+}^{N \backslash i}$, the slope of $\xi_{i}\left(\lambda, x_{-i}\right)$ at $\lambda=x_{i}$ lies between $F_{-}^{\prime}\left(\delta_{i}\left(x_{i}\right)\right)$ and $F_{+}^{\prime}\left(x_{i}\right)$. It follows from the strict concavity of $\xi_{i}\left(\cdot, x_{-i}\right)$ that $x_{i}$ maximizes $u_{i}^{*}\left(\lambda, \xi_{i}\left(\lambda, x_{-i}\right)\right)$ on $\mathbb{R}_{+}^{N \backslash i}$ for any $x_{-i} \in \mathbb{R}_{+}^{N \backslash i}$, completing the proof of the lemma. 
Figure 1
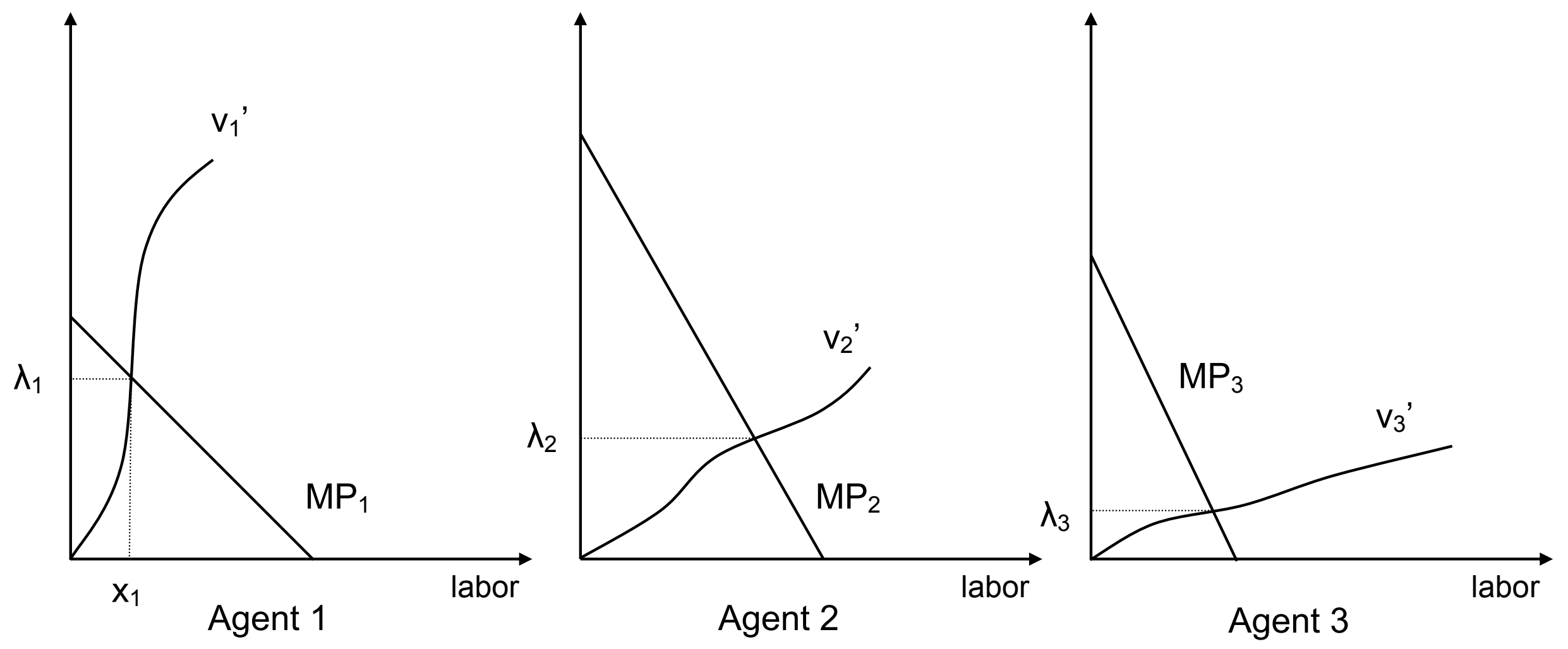
Figure 2

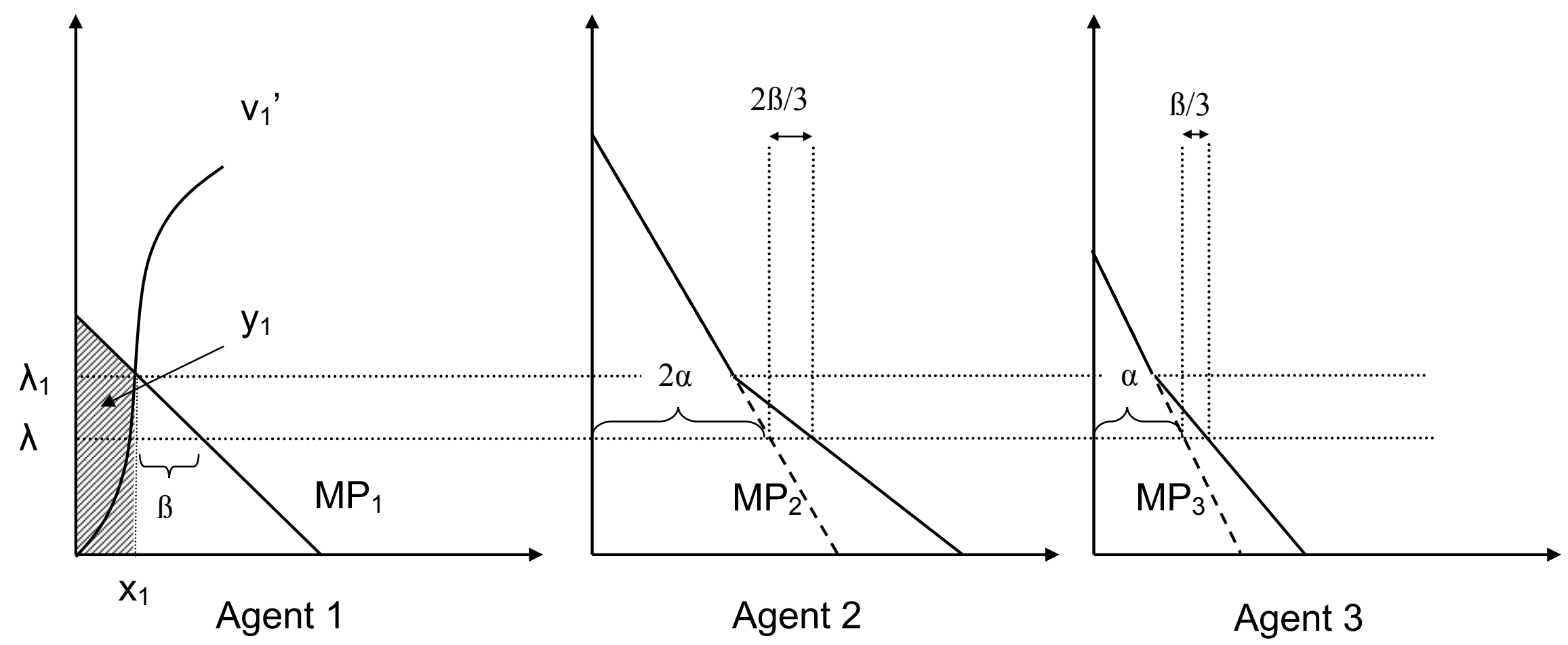


Figure 3

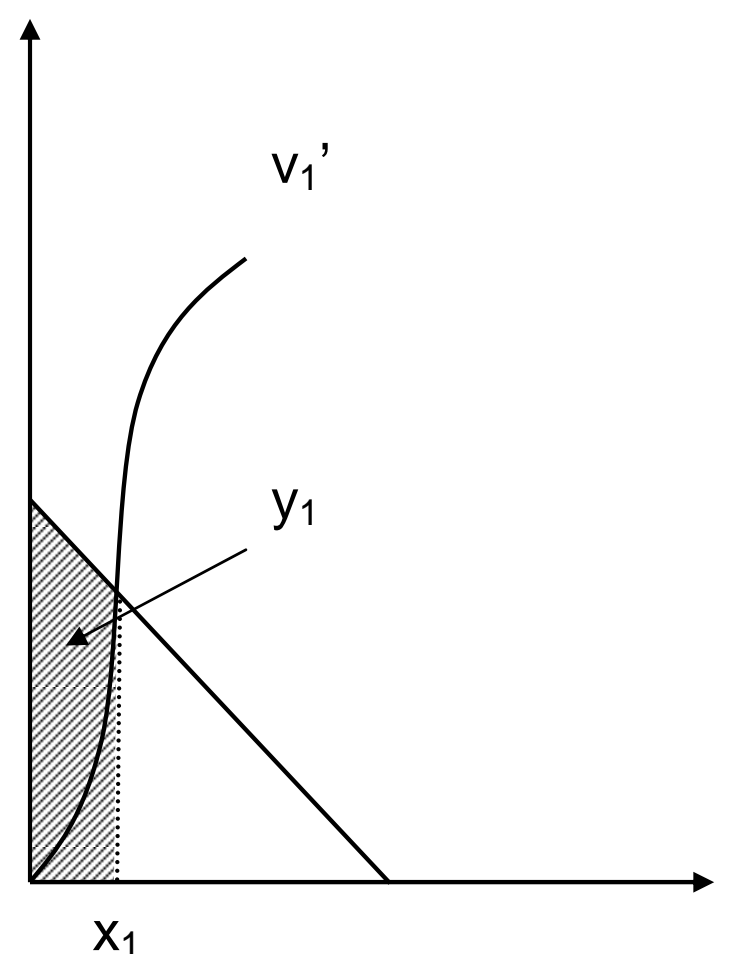

Agent 1

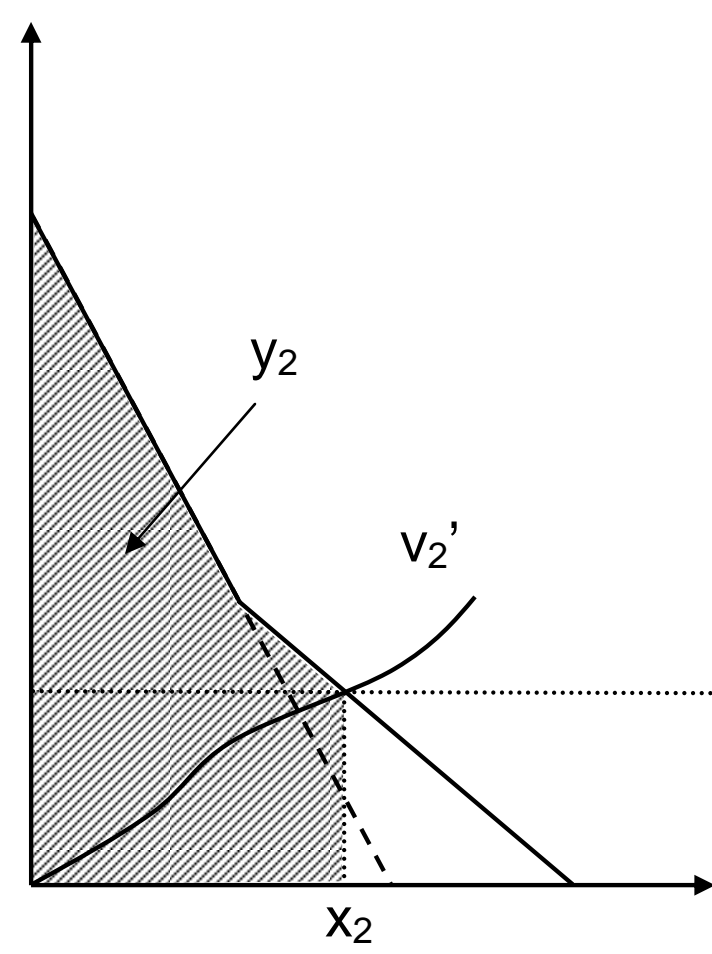

Agent 2

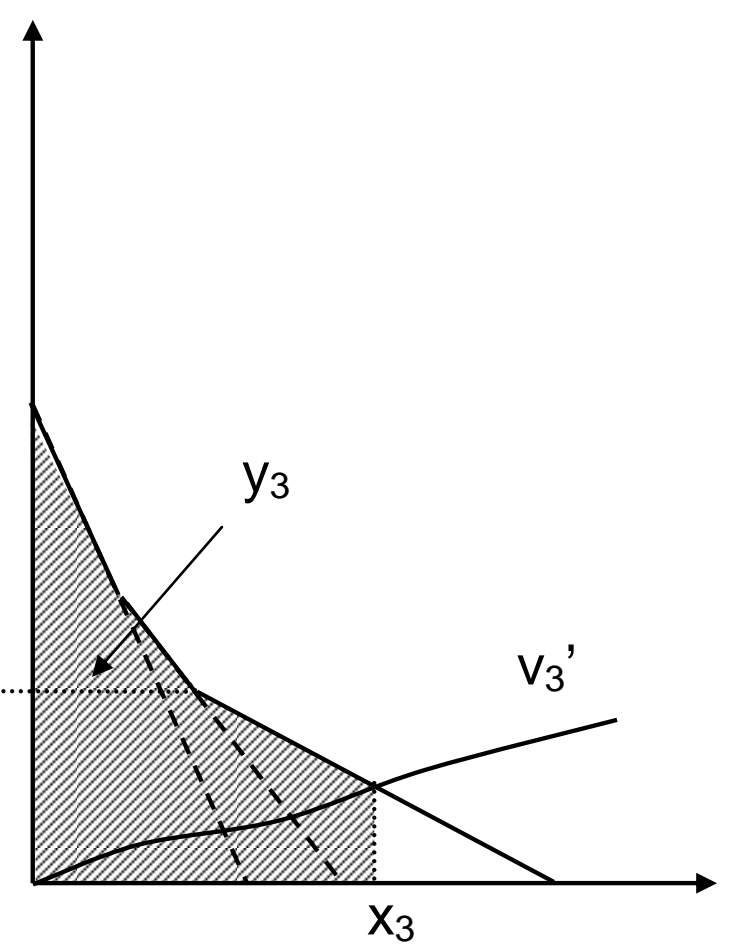

Agent 3 


\section{References}

[1] Corchón LC, Puy MS (2002) Existence and Nash implementation of efficient sharing rules for a commonly owned technology. Soc. Choice Welfare 19: 369-379

[2] Craig B, Pencavel J (1995) Participation and Productivity: A comparison of worker cooperatives and conventional firms in the plywood industry. Brookings Pap. Econ. Act. Microeconomics vol. 1995: 121-174

[3] Dasgupta P, Hammond P, Maskin E (1979) The implementation of social choice rules: Some results on incentive compatibility. Rev. Econ. Stud. 46: $185-216$

[4] Friedman EJ(2002) Strategic properties of heterogeneous serial cost sharing. Math. Soc. Sci. 44: 145-154

[5] Friedman EJ(2004) Strong monotonicity in surplus sharing. Econ. Theory 23: $643-658$

[6] Gilson R, Mnookin R (1985) Sharing Among the Human Capitalists: An Economic Inquiry into the Corporate Law Firm and How Partners Share Profits. Stanford Law Review 37:313-392

[7] Israelsen D.(1980) Collectives, Communes, and Incentives. J. Compar. Econ. 4: $99-124$

[8] Leroux J (2004) Strategyproofness and efficiency are incompatible in production economies. Econ. Letters 85: 335-340.

[9] Leroux J (2005) Strategy-proof surplus sharing: a 2-agent characterization. mimeo, Rice University, 2005

[10] Moulin H (1999) Incremental cost sharing: Characterization by coalition strategyproofness. Soc. Choice Welfare 16: 279-320

[11] Moulin H, Shenker S (1992) Serial Cost Sharing Econometrica 60:10091037

[12] Oppel RA Jr.(2003) Panel Finds Manipulation by Energy Companies. New York Times March 27 
[13] Saijo T (1991) Incentive compatibility and individual rationality in public good economies. J. Econ. Theory 55:203-212

[14] Sen AK (1966) Labour allocation in a cooperative enterprise. Rev. Econ. Stud. 33:361-371

[15] Shin S,.Suh SC (1997) Double implementation by a simple game form in the commons problem. J. Econ. Theory 77: 205-213

[16] Sprumont Y.(1998) Ordinal Cost Sharing. J. Econ. Theory 81:126-162

[17] Townsend RE (1995) Fisheries self-governance: corporate or cooperative structures Marine Policy 19: 39-45

[18] Weitzman M (1974) Free access vs private ownership as alternative systems for managing common property. J. Econ. Theory 8: 225-234. 


\section{Liste des cahiers de recherche publiés par les professeurs 2005-2006}

\section{Institut d'économie appliquée}

IEA-05-01 DÉSIRÉ VENCATACHALLUM ET BRUNO VERSAEVEL. « R\&D Delegation in a Duopoly with Spillovers », 57 pages.

IEA-05-02 MiCHEL NORMANDIN ET PASCAL ST-AMOUR.. « An Empirical Analysis of U.S. Aggregate Portfolio Allocations », 33 pages.

IEA-05-03 MARTIN BOILEAu ET MiCHEL nORMANDin. « Closing International Real Business Cycle Models with Restricted Financial Markets », 36 pages.

IEA-05-04 GEORGES DIONNE ET BENOIT DOSTIE. «New Evidence on the Determinants of Absenteeism Using Linked Employer-Employee Data », 35 pages.

IEA-05-05 MAURICE N. MARCHON. «Perspectives économiques canadiennes dans un contexte international », 27 pages.

IEA-05-06 RAYNAULD, JACQUES. «L'efficacité de l'apprentissage en ligne: le cas d'un cours d’économie à HEC Montréal », 10 pages

IEA-05-07 MICHEL NORMANDIN ET BRUNO POWO FOSSO. «Global versus Country-Specific Shocks and International Business Cycles», 44 pages.

IEA-05-08 RAYNAULD, JACQUES. « Le programme Virtuose : les ordinateurs portatifs et l'intégration des TIC à HEC Montréal - 1996-2001 », 88 pages 
IEA-06-01 DOSTIE, BENOIT ET LÉGER PIERRE THOMAS. « Self-selection in migration and returns to unobservable skills », 88 pages

IEA-06-02 JÉRÉMY LAURENT-LUCCHETTI AND ANDREW LEACH. «Induced innovation in a decentralized model of climate change », 34 pages.

IEA-06-03 BENOIT DOSTIE, RAJSHRI JAYARAMAN AND MATHIEU TRÉPANIER. « The Returns to Computer Use Revisited, Again », 27 pages.

IEA-06-04 MICHEL NORMANDIN. « The Effects of Monetary-Policy Shocks on Real Wages: A MultiCountry Investigation », 38 pages.

IEA-06-05 MICHEL NORMANDIN. «Fiscal Policies, External Deficits, and Budget Deficits », 50 pages.

IEA-06-06 J. DAVID CUMMINS, GEORGES DIONNE, ROBERT GAGNÉ AND ADBELHAKIM NOUIRA. «Efficiency of Insurance Firms with Endogenous Risk Management and Financial Intermediation Activities », 41 pages.

IEA-06-07 LUC BAUWENS AND JEROEN V.K. ROMBOUTS. «Bayesian Inference for the Mixed Conditional Heteroskedasticity Model », 25 pages.

IEA-06-08 LUC BAUWENS ARIE PREMINGER AND JEROEN V.K. ROMBOUTS. «Regime Switching Garch Models », 25 pages.

IEA-06-09 JEROEN V.K. ROMBOUTS AND TAOUFIK BOUEZMARNI. « Nonparametric Density Estimation for Positive Time Series », 32 pages. 